\title{
Evaluation of Left Ventricular Diastolic Function Using 4D Flow MR Imaging
}

\author{
Sophia Houriez--Gombaud-Saintonge ${ }^{1,2}$, Emilie Bollache ${ }^{1}$, Gilles Soulat ${ }^{3}$, Youssef Alattar ${ }^{3}$, \\ Jérôme Lamy ${ }^{1}$, Thomas Dietenbeck ${ }^{1}$, Alain De Cesare ${ }^{1}$, Alain Giron ${ }^{1}$, Kevin Bouaou ${ }^{1}$ Umit \\ Gencer $^{3}$, Alban Redheuil ${ }^{1}$, Elie Mousseaux ${ }^{3}$, Yasmina Chenoune ${ }^{2}$, Nadjia Kachenoura ${ }^{1}$ \\ ${ }^{1}$ Sorbonne Université, INSERM, CNRS, Laboratoire d'Imagerie Biomédicale, Paris, France \\ ${ }^{2}$ ESME SUDRIA, ESME Research Lab, Paris, France \\ ${ }^{3}$ Hopital Européen Georges Pompidou, Paris, France
}

\begin{abstract}
Aim: to 1) evaluate the ability of $4 D$ flow MRI to assess left ventricular (LV) diastolic function, as compared to ultrasound (US), 2) analyse simultaneously LV filling and aortic stiffness in aging. Method: We studied 36 healthy subjects (52 \pm 18 years) who underwent MRI and US exams. A custom software was used for LV segmentation and calculation of transmitral flow-rate curves in a $4 D$ flow MRI stack of slices located between the mitral valve and the apex and perpendicular to the LV long axis. Finally, the early (E) and late (A) flow-rate peaks were automatically detected and the E/A ratio was computed. Aortic pulse wave velocity $(P W V)$ was also calculated from the 4D flow images. Results: A significant and high correlation was found between $4 D$ flow and US E/A $(R=0.71, p<0.001)$. Besides, $a$ higher correlation was obtained between E/A and age when using $4 D$ flow than US (E/A vs. age: $R_{4 D}=-0.87$, $\left.p<0.001, R_{U S}=-0.69, p<0.001\right)$. Thanks to $4 D$ flow MRI, simultaneous variation of aortic $P W V$ and diastolic function with age was further illustrated. Conclusion: 4D flow MRI provided consistent LV E/A measurements in terms of comparison against reference $U S$ and associations with age. It also provided an insight into aorta/left heart coupling.
\end{abstract}

\section{Introduction}

The prevalence of heart failure is unceasingly increasing with population ageing, reaching at least 26 million affected people in the world [1]. Left ventricular (LV) ejection fraction is the most widely used clinical biomarker to evaluate LV function. However, previous studies have shown that 40 to $50 \%$ of patients had heart failure whereas their LV ejection fraction remained preserved [2]. Clinical guidelines in echocardiography highlighted the usefulness of mitral inflow measurement and longitudinal velocity of the mitral annulus [3] to assess LV diastolic function and subsequently help for the characterization of patients with heart failure.
The recent 4D flow MRI sequence offers an excellent anatomical coverage and allows measuring blood flow velocities in the three spatial directions through time within a $3 \mathrm{D}$ volume. Such data allow studying the aorta and the left heart (LV and left atrium) during a single acquisition ensuring their evaluation under the same hemodynamic conditions. Several studies reported the ability of 4D flow MRI to accurately assess aortic hemodynamics either locally by estimating wall shear stress [4] or globally by estimating aortic pulse wave velocity (PWV) [5].

Our objectives were: 1) to achieve a quantitative analysis of transmitral inflow from 4D flow MRI data to evaluate diastolic function as compared to echocardiography, 2) to simultaneously evaluate aortic and LV alterations in aging and provide a preliminary insight into their overall trends in a population of 36 healthy volunteers with a uniform distribution of age between 20 and 80 years.

\section{Material and methods}

\subsection{Population and data acquisition}

We included 36 healthy subjects aged from 20 to 80 years (52 \pm 18 years), free from overt cardiovascular disease. The protocol has been approved by the local ethics committee and all participants signed an informed consent.

All subjects underwent an MRI exam on a 3T magnet (GE Healthcare) during free breathing with prospective ECG gating. 4D flow was acquired in both the LV and the aorta using the following scan parameters: velocity encoding $=250 \mathrm{~cm} / \mathrm{s}$, echo time $=1.7 \mathrm{~ms}$, repetition time $=4.3-4.4 \mathrm{~ms}$, flip angle $=15^{\circ}$, voxel size $=1 \times 1.48 \times 2.38 \mathrm{~mm}^{3}, 50$ timephases. To enhance the signal to noise ratio, a single dose of contrast agent was used. In addition, conventional $\mathrm{LV}$ cine images were acquired and analysed to derive LV mass, end systolic (ESV), end diastolic (EDV) volumes and ejection fraction (EF). Phase offset correction and velocity de-aliasing of 4D flow images were applied. In addition, aortic data 


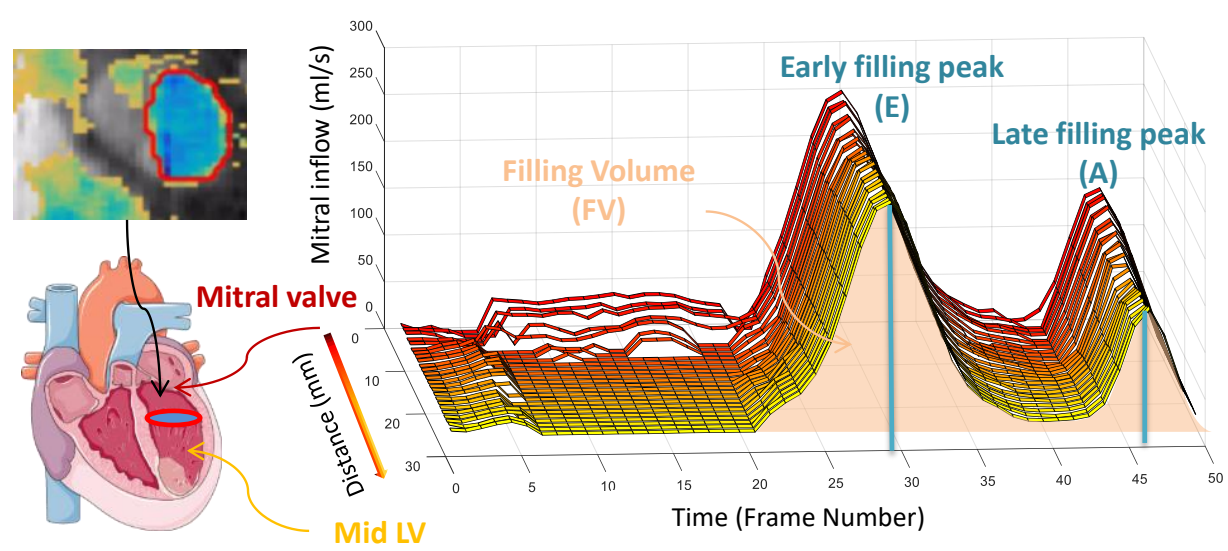

Figure 1. 4D flow MRI transmitral flow-rate curves calculated between the mitral annulus and mid $\mathrm{LV}$, along with the extracted peaks (E, A) and filling volume (FV)

were analysed to estimate the aortic stiffness-related PWV, using flow curves extracted from planes perpendicular to the aortic centerline throughout the entire aorta [5]. Briefly, PWV was calculated as the linear regression slope of aortic segment lengths vs. transit times needed to travel such distances. Transit time was successively calculated between the flow curve in the distal descending aorta and each flow curve extracted along the aortic centerline up to the ascending aorta. Such time shift calculation was based on the wavelet method.

All subjects also underwent an echocardiography exam on the same day and transmitral flow early (E) and late (A) peak velocities were measured along with the maximal mitral annulus longitudinal velocity (E'). E/A and E/E' ratios were calculated.

\subsection{Transmitral inflow segmentation}

To segment the LV, we adapted an existing algorithm developed for 2D phase contrast MRI [6] based on the connectivity of pixels in terms of velocity sign, and implemented the following steps into a custom software (Matlab, The Mathworks, Natick, MA). First, a rough transmitral flow velocity curve was calculated in a manually positionned marker within the LV base, to select the LV early filling time phase. Second, two anatomical landmarks namely the mitral valve and the apex were manually positionned to define the LV long axis. Then, the short axis slice at the level of the mitral annulus was displayed to draw a rough region of interest (ROI) around the transmitral flow, used to initialize the segmentation process. The time-resolved mean velocity curve was claculated within such ROI and used to define the correct phase corresponding to peak velocity. On this peak velocity phase, the largest connected area in terms of velocity sign (inflow) was automatically detected and its barycentre was calculated. This barycentre was then propagated to neighboring time phases, and the largest connected area in terms of velocity sign surrounding it was detected. Such process was repeated towards the beginning and the end of the cardiac cycle. The largest ROI on this most basal slice was propagated to the following slice to perform the time propagation segmentation process. Finally, This latter step was repeated on the next slices along the LV long axis until the apical region was reached, while initializing using the largest ROI obtained on the previous slice.

\subsection{Blood flow functional parameters}

The following parameters were automatically derived from the flow-rate curves extracted from transmitral inflow segmentation of 4D flow images (Fig. 1): 1) E (ml. $\left.\mathrm{s}^{-1}\right)$ wave peak, defined as the first transmitral flow-rate curve maximum; 2) A (ml.s $\left.\mathrm{s}^{-1}\right)$ wave peak, defined as the second transmitral flowrate curve maximum; 3) E/A calculated as the ratio between the early and the late filling peaks; 4) E/FV $\left(\mathrm{s}^{-1}\right)$ calculated as the ratio between the early filling peak and the total filling volume (FV-ml), computed as the area under the transmitral flow-rate curve and 5) $\mathrm{A} / \mathrm{FV}\left(\mathrm{s}^{-1}\right)$, calculated as the ratio between the late filling peak and $\mathrm{FV}$.

These parameters were computed for all LV short axis slices comprised between the mitral annulus and mid-LV along the LV long axis. Then, for each parameter, we considered the value of the $9^{\text {th }}$ decile.

Of note, only flow-rate based measurements were presented because they were more reliable than maximal velocities for 4D flow MRI. Indeed, maximal velocity is strongly affected by the encoding velocity set during acquisition and the subsequent noise. In addition, peak flow rate indices were normalized by the total filling volume to account for difference in flow load conditions among individuals. 


\subsection{Statistical analysis}

All quantitative indices were provided as means \pm standard deviations (SD). Linear regression using Spearman correlation was used to study relationships between MRI diastolic function indices and age, aortic PWV and echocardiographic E/A ratio. Bland-Altman analysis was performed for comparisons of 4D flow against echocardiographic $\mathrm{E} / \mathrm{A}$; mean bias and confidence interval (CI) (mean bias $\pm 1.96 \times \mathrm{SD})$ were provided. Statistical analyses were performed using JMP and Matlab.

\section{Results and discussion}

Table 1 summarizes subjects characteristics along with cine MRI LV indices as well as transmitral flowrelated diastolic function parameters measured with echocardiography and 4D flow MRI. Our subjects had normal LV size and ejection fraction, and the E/A ratio was on average in the same range when measured by echocardiography and 4D flow MRI [7]. Echocardiographic and MRI E/A ratios were further strongly correlated $(\mathrm{R}=0.71, \mathrm{p}<0.0001)$ and the Bland-Altman mean bias and CI between the two measurements were reasonable $(-0.01 \pm 0.82)$. Although a direct comparison was not possible because of differences in methods and populations, correlations obtained in this study for comparison against echocardiography were consistent with previous 2D phase contrast MRI studies [6].

\begin{tabular}{|c|c|c|c|}
\hline & \multirow{2}{*}{ Mean \pm std } & \multicolumn{2}{|c|}{$\begin{array}{l}\text { Associations } \\
\text { with age }\end{array}$} \\
\hline & & $\mathrm{R}$ & $\mathrm{p}$ \\
\hline \multicolumn{4}{|c|}{ Subjects characteristics } \\
\hline Age (years) & $51.6 \pm 17.5$ & - & - \\
\hline BMI $\left(\mathrm{kg} / \mathrm{m}^{2}\right)$ & $23.5 \pm 2.8$ & 0.4 & 0.02 \\
\hline $\mathrm{EF}(\%)$ & $62.1 \pm 5.1$ & 0.07 & 0.69 \\
\hline LV mass $(\mathrm{g})$ & $94.6 \pm 25.1$ & -0.08 & 0.660 \\
\hline LV mass/BSA $\left(\mathrm{g} / \mathrm{m}^{2}\right)$ & $53.2 \pm 10.5$ & -0.24 & 0.15 \\
\hline $\operatorname{LV} \operatorname{EDV}\left(\mathrm{ml} / \mathrm{m}^{2}\right)$ & $74.5 \pm 17$ & -0.55 & 0.001 \\
\hline $\operatorname{LV} \operatorname{ESV}\left(\mathrm{ml} / \mathrm{m}^{2}\right)$ & $28.3 \pm 7.5$ & -0.49 & 0.003 \\
\hline \multicolumn{4}{|c|}{ Echocardiographic measurements } \\
\hline $\mathrm{E} / \mathrm{A}$ & $1.2 \pm 0.4$ & -0.69 & $<0.001$ \\
\hline $\mathrm{E}(\mathrm{cm} / \mathrm{s})$ & $71.5 \pm 16.9$ & -0.19 & 0.25 \\
\hline $\mathrm{A}(\mathrm{cm} / \mathrm{s})$ & $61.8 \pm 18.8$ & 0.67 & $<0.001$ \\
\hline $\mathrm{E} / \mathrm{E}^{\prime}$ & $5.6 \pm 2.3$ & 0.65 & $<0.001$ \\
\hline \multicolumn{4}{|c|}{ 4D Flow MRI measurements } \\
\hline E/A & $1.2 \pm 0.6$ & -0.87 & $<0.001$ \\
\hline $\mathrm{E} / \mathrm{FV}\left(\mathrm{s}^{-1}\right)$ & $3.6 \pm 1.1$ & -0.63 & $<0.001$ \\
\hline $\mathrm{A} / \mathrm{FV}\left(\mathrm{s}^{-1}\right)$ & $3.3 \pm 0.8$ & 0.61 & $<0.001$ \\
\hline $\mathrm{PWV}(\mathrm{m} / \mathrm{s})$ & $8.6 \pm 3.2$ & 0.82 & $<0.001$ \\
\hline
\end{tabular}

Table 1. Subjects characteristics with echocardiographic and MRI measurements

\subsection{Associations with age}

Associations between the measured LV diastolic function indices and age are shown in Table 1. As expected, echocardiographic $\mathrm{E}$ and MRI E/FV decreased with age. However, only the MRI index provided significant association with age. Both echocardiographic A and MRI A/FV consistently and significantly increased with age as the left atrial (LA) booster function increases with age, with a slightly higher correlation coefficient for echocardiography. Finally, the E/A ratio significantly decreased with age for both modalities in agreement with various echocardiographic and MRI studies, with a higher correlation coefficient for MRI. (MRI E/A: $\mathrm{R}=-0.87$, $\mathrm{p}<0.001$; echocardiography $\mathrm{E} / \mathrm{A}: \mathrm{R}=-0.69, \mathrm{p}<0.001)$. In line with the increase in LV filling pressures with aging, the echocardiographic $\mathrm{E} / \mathrm{E}$ ' ratio increased significantly with age. Such index was not calculated from 4D flow MRI, because the encoding velocity was tuned for imaging blood flow and was thus too high to be able to estimate non-noisy myocardial velocities. An alternative would be to estimate E' from cine MRI data by using feature tracking of the mitral annulus longitudinal motion.

\subsection{Association with PWV}

Since aortic stiffness indices are associated with $\mathrm{LV}$ remodeling independent of age, we would expect an association between the LV E/A ratio with aortic PWV. Indeed, LV remodeling in aging would affect LV wall stiffness and thus $\mathrm{LV}$ filling pressures affecting the LV early and late filling.

MRI measurements were significantly associated with aortic PWV: E/A ( $\mathrm{R}=-0.71, \mathrm{p}<0.001)$, E/FV $(\mathrm{R}=-0.41, \mathrm{p}=0.01)$ and $\mathrm{A} / \mathrm{FV}(\mathrm{R}=0.65, \mathrm{p}<0.001)$. However, such associations were no longer significant after adjustment for age.

\subsection{Mixed effects of aging and stiffness}

Such association with aortic PWV through age is illustrated in Figure 2, which shows an increase in aortic PWV that follows the linear variation of diastolic function indices with age. This complex and inconclusive association between age, LV diastolic function indices and aortic stiffness might be explained by the "normal" filling pressures of our subjects, as measured by the echocardiographic E/E'. Indeed only 4 subjects had an $\mathrm{E} / \mathrm{E}$ ' ratio above 8 and none had E/E' ratio above 14 . 

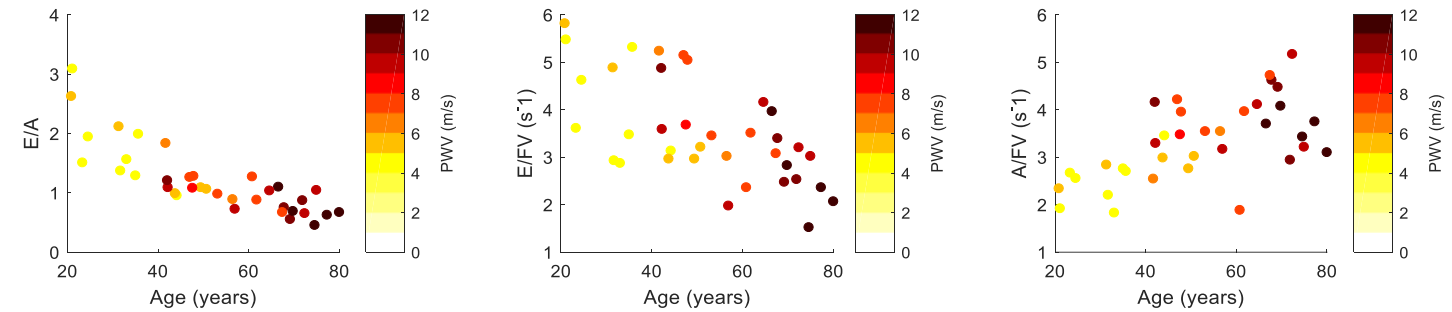

Figure 2. Associations between MRI LV E/A, E/FV, A/FV (FV: filling volume) and age. Graph dots are colorcoded according to their 4D flow MRI-derived aortic pulse wave velocity (PWV) values.

4D flow MRI enabled a simultaneous evaluation of aortic stiffness, through PWV, the LV diastolic filling, through E/A, and the LA booster function using $\mathrm{A} / \mathrm{FV}$. To compare the age-related variations of such indices, we first normalized them relative to their maximal value to account for the differences in their magnitudes and units. Associations of such normalized values with age are shown in Figure 3. This graphical illustration shows a parallel variation of E/A and aortic PWV with age (0.8-0.9\%/ year) and a slightly slower age-related progression of $\mathrm{A} / \mathrm{FV}$ $(0.6 \% /$ year). This might be explained by the perpetual coupling between the aorta and LV (cardiac cycle, afterload condition ...), while the LA might be protected to some extent through complex mechanical and hemodynamic phenomena induced by LV remodeling. Such results should be interpreted with caution because of the small sample size and the differences in physiological and hemodynamic meaning of the considered indices.

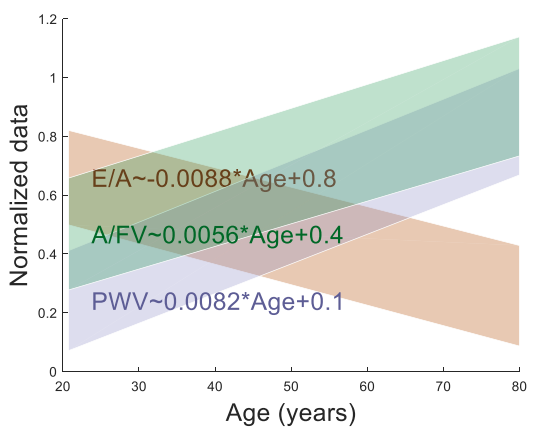

Figure 3. Linear regression of normalized aortic PWV, E/A, and A/FV with age. Shaded colours illustrate confidence intervals.

\section{Conclusion}

4D flow MRI was successfully used to quantify diastolic transmitral inflow as demonstrated by the strong associations with reference Doppler echocardiography and by the expected decline in the extracted indices with age. The application of the methods to simultaneously evaluate aortic PWV and
LV diastolic function presented in this work might be useful for a better understanding of the processes of aging and coupling of the left heart and the aorta.

\section{Acknowledgements}

We would like to acknowledge the FRM project ING20150532487 and ESME SUDRIA.

\section{References}

[1] Savarese G, Lund LH. Global Public Health Burden of Heart Failure. Card Fail Rev 2017; 3: 7-11.

[2] Zile MR, Baicu CF, Gaasch WH. Diastolic heart failure-abnormalities in active relaxation and passive stiffness of the left ventricle. N Engl J Med 2004; 350: 1953-1959.

[3] Nagueh SF, Smiseth OA, Appleton CP, et al. Recommendations for the Evaluation of Left Ventricular Diastolic Function by Echocardiography: An Update from the American Society of Echocardiography and the European Association of Cardiovascular Imaging. Eur Heart J Cardiovasc Imaging 2016; 17: 1321-1360.

[4] Bollache E, Guzzardi DG, Sattari S, et al. Aortic valvemediated wall shear stress is heterogeneous and predicts regional aortic elastic fiber thinning in bicuspid aortic valve-associated aortopathy. J Thorac Cardiovasc Surg. 2018. DOI: 10.1016/j.jtcvs.2018.05.095.

[5] Houriez-Gombaud-Saintonge S, Mousseaux E, Bargiotas I, et al. The assessment of aortic pulse wave velocity using 4D flow magnetic resonance imaging: Methods comparison. Computing in Cardiology. 2017, pp. 1-4.

[6] Bollache E, Redheuil A, Clément-Guinaudeau S, et al. Automated left ventricular diastolic function evaluation from phase-contrast cardiovascular magnetic resonance and comparison with Doppler echocardiography. J Cardiovasc Magn Reson 2010; 12: 63.

[7] Kawel-Boehm N, Maceira A, Valsangiacomo-Buechel ER, et al. Normal values for cardiovascular magnetic resonance in adults and children. $J$ Cardiovasc Magn Reson 2015; 17 : 29.

Address for correspondence.

Sophia Houriez--Gombaud-Saintonge.

15 rue de l'école de médecine 75006, Paris

sophia.houriez--gombaud-saintonge@etu.upmc.fr 